\title{
Las floristerías, microempresas generadas como emprendimientos en base a la normativa legal.
}

Florists, micro-businesses generated as enterprises based on legal regulations.

Diego Patricio Vallejo Sánchez. ${ }^{1}$, Raquel Virginia Colcha Ortiz. ${ }^{2} \&$ María del Carmen Moreno Albuja. ${ }^{3}$

\begin{abstract}
.
DOI: https://doi.org/10.33262/concienciadigital.v3i2.2.1244

The research synthesizes the results of surveys carried out on a whole segment of commerce in the city of Riobamba, in the research it was developed as a cross-sectional, descriptive and analytical study, it was analyzed to the people who commercialize or produce flower arrangements, that in the city are 53, the objective was to determine if they emerged as entrepreneurship and according to the business turn can be considered as micro-enterprises, at the time prior to the surveys were explained to the unit of study what is a venture and what is a microenterprise, as well as the differences that exist between them, A business arises from the business idea that a person intends to carry out to generate their own income as well as not depend on a third party. Micro, small and medium enterprises are those that generate the highest income for a country, because these businesses have been created according to the entrepreneur's skills, in Among the determinant results of the research, it was determined that the total number of businesses emerged as undertakings, and in addition, $66 \%$ of the respondents stated that their businesses could be considered as micro-enterprises.
\end{abstract}

Keywords. Micro-Enterprises, flower, Entrepreneurship, Legal Regulations

\section{Resumen.}

La investigación fue realizada en la ciudad de Riobamba y sintetiza los resultados de encuestas realizadas a todo un segmento del comercio de la misma, en la investigación se la desarrolló como un estudio transversal, descriptivo y analítico, se analizó a las personas que comercializan o producen arreglos florales, que en la ciudad son 53 en base a información obtenida por el GAD cantonal, el objetivo fue determinar si las mismas surgieron como emprendimientos y de acuerdo al giro del negocio se pueden considerar como microempresas, en el momento previo

\footnotetext{
${ }^{1}$ Escuela Superior Politécnica de Chimborazo, Facultad de Administración de Empresas Chimborazo Ecuador, diego_vallejo@espoch.edu.ec

${ }^{2}$ Escuela Superior Politécnica de Chimborazo, Facultad de Administración de Empresas Chimborazo Ecuador, raquel.colcha@espoch.edu.ec

${ }^{3}$ Escuela Superior Politécnica de Chimborazo, Facultad de Administración de Empresas Chimborazo Ecuador, carmen.moreno@espoch.edu.ec
} 
a realizarse las encuestas se explicó a la unidad de estudio qué es un emprendimiento y qué es una microempresa, así como también las diferencias que existen entre las mismas, Un emprendimiento surge de la idea de negocio que una persona pretende llevar a cabo para generar ingresos propios así como no depender de un tercero, Las micro, pequeñas y medianas empresas son las que generan los mayores ingresos para un país, pues dichos negocios se han creado de acuerdo a las habilidades del emprendedor, entre los resultados determinantes de la investigación se determinó que la totalidad de comercios surgieron como emprendimientos y además el $66 \%$ de los encuestados manifiestan que sus negocios se podrían considerar como microempresas.

Palabras Claves. Microempresas, Flor, Emprendimientos, Normativa Legal

\section{Introducción.}

En los últimos años el emprendimiento se ha convertido en un elemento primordial en el desarrollo económicos de un sector específico, debido al incremento de la productividad, es decir permite tener diferentes iniciativas que generan riqueza o movimiento de dinero tanto de la cuidad, país e incluso promueve el factor humano a que este tienda a crecer a gran nivel. Un emprendimiento requiere de la iniciativa del ser humano para poder salir hacia adelante, en la búsqueda de diversas maneras de encontrar éxito y obtener ingresos propios del desarrollo de la idea económica. La cuidad de Riobamba, ha sido una ciudad en donde sus habitantes han planteado ideas de emprendimientos propios y a su vez estos han sido aportes para la economía local. Debido a esto se ha visto la presencia de nuevas microempresas de cualquier sector entre ellos se puede palpar con mayor facilidad, negocios de floristería, restaurants, empresas textiles entre otros.

Con este artículo se pretende dar a conocer que se entiende por emprendimiento y a su vez un estudio del sector florícola de la cuidad, para entender y comprender como la idea de emprendimiento con los años ha tomado fuerza, permitiendo así tener gran cantidad de locales dedicados a esta actividad. A su vez como han crecido a lo largo del tiempo y a la vez encontrar las dificultades o debilidades que pueden tener así como también las deficiencias que presentan, todo esto para entender cómo se maneja un negocio propio que permite generar ingresos propios sin depender de terceras personas que controlen el tiempo de trabajo y la forma de hacerlo.

Para el desarrollo del presente artículo también se ha visto la necesidad de incurrir en ciertos libros en los que se definen términos indispensables para la investigación.

Entre los principales se enuncian los siguientes:

\section{Mipyme.}

Según Ameconi (2004), en su libro Microempresas en Acción, manifiesta que las MIPYMES en muchos países del mundo constituyen el centro de la actividad de la economía; en América Latina y el Caribe las empresas que tienen 10 empleados o menos generan entre el 30 y $80 \%$ de los empleos, que las 50 millones de microempresas que existen en la región dan empleos variados a más de 150 millones de personas. Es necesario notar que la mayoría de los dueños 
y empleados de estas empresas son personas de bajos niveles de ingresos, tienen una educación formal elemental y el subempleo es frecuente, especialmente en el caso de las mujeres. Las medianas, pequeñas y las micro empresas, constituyen uno de los pilares fundamentales de nuestra economía y de América Latina; sin desmerecer el aporte que dan las grandes empresas, ignorarlas, marginarlas o no darles el apoyo que requieren las MIPYMES, será siempre contraproducente para el desarrollo económico, es por ello que los problemas que condicionan su funcionamiento, su eficiencia ha sido motivo de preocupación constante de los gobiernos y en mayor medida de los investigadores.

Una MIPYME se la conoce como micro, mediana y pequeña empresa, de acuerdo a lo que establece o reconoce el ministerio de industrias de Ecuador. En nuestro país las pequeñas y medianas empresas son importantes ya que representan un aporte importante a la economía ecuatoriana por ello se han desarrollado varias campañas que benefician a los emprendedores de las pequeñas y medianas empresas dándoles créditos, y extinguiendo impuestos a los emprendedores que recién están empezando con un negocio que aporte al crecimiento de la economía ecuatoriana.

Con frecuencia se ha podido establecer que las pequeñas empresas son la base de las empresas grandes ya que estas ofrecen sus servicios o bienes para las grandes empresas y también estas pueden llegar a convertirse en grandes empresas brindado más oportunidades de trabajo para los ecuatorianos.

\title{
Emprendimiento
}

El emprendimiento es un término muy utilizado en la actualidad es un término que ha estado presente en la humanidad a lo largo de la historia. Como lo señala Borras, et al (2017), en su obra El emprendimiento: una aproximación internacional al desarrollo económico: " $L a$ mayoría de los estudios sobre emprendimiento, han analizado el proceso de creación de empresas desde una perspectiva centrada en los principales rasgos y características que se deben fomentar en el emprendedor para garantizar el éxito del proyecto". El emprendimiento es el estudio de como las empresas han sido creadas por ello es necesario que se conozcan los emprendedores de cómo realizar un correcto estudio del mercado para que el emprendimiento que se pretenda realizar tenga os beneficios que esperan las personas que han decidido crear el negocio.

\section{Tipos de emprendimiento}

Según Schnarch (2014), en su texto indica dos tipos de emprendimiento y son los siguientes:

\author{
Intrapreneur \\ "Se entiende por intrapreneur a los empresarios que están dentro de la empresa son \\ quienes asumen la responsabilidad activa de producir en la empresa" \\ Entrepreneur \\ "Entendemos por Entrepreneur a un empresario independiente busca crear empresas"
}




\section{Emprender}

Según Alcaraz (2011), en su texto manifiesta que emprender "es un vocablo que denota un perfil, un conjunto de características que hacen actuar a una persona de una manera determinada y le permiten mostrar ciertas competencias para visualizar, definir y alcanzar objetivos".

Sin embargo esta no es la única definición que se tiene para este término, pues existe muchos catedráticos o personas de la rama que dan un significado diferente pero manteniéndose en la misma línea de análisis, solo que según su óptica y experiencia lo catalogan con otro términos mucho más comprensibles para el público lector.

\section{Emprendedor}

Se define como emprendedor según Schnarch (2014): "Agente que compra los medios de producción a ciertos precios y los combina en forma ordenada para obtener de allí en un nuevo producto" es decir es la persona que produce un bien para ofrecerlo al público sin embargo el emprendedor se diferencia de otros agentes por no poseer un retorno seguro es decir que es el quien invierte con el riesgo de no obtener el beneficio esperado

El emprendedor se relaciona con varias disciplinas tales como se definen en Schnarch (2014): "Economía, estrategias de negocio, Comportamiento organizacional, Sociología y Psicología. Los economistas definen al emprendedor en función de las actividades y los logros conducentes a afectar el sistema económico; los sociólogos buscan comprender el modo en el que el comportamiento de los emprendedores tienen lugar en la sociedad y puede afectarla, y los psicólogos se focalizan en las características personales y el proceso cognitivo de esto" Es decir, que el comportamiento del emprendedor es estudiado por los economistas, sociólogos y psicólogos con el fin de conocer como este actúa en el mercado al momento de ofrecer un producto.

En el ecuador existen vendedores informales y la pregunta es ¿los informales son emprendedores? A los que se responde que estos vendedores tienen la idea para crear un negocio el problema es que en diversas ocasiones no han encontrado la manera de plasmar su idea convirtiéndola en micro empresa y en efecto podemos definir al vendedor informal como un pequeño emprendedor quien con esfuerzo y al encontrar los métodos y técnicas este puede convertirse en empresario.

\section{Características del emprendedor}

Existe ciertas características que permiten distinguir a un emprendedor de cualquier otra persona y es así como Alcaraz (2011), en su texto manifiesta lo siguiente:

- Posee un compromiso total, perseverancia y responsabilidad.

- Tiene una capacidad para alcanzar las metas planteadas.

- Tiende a perseguir sus objetivos y metas.

- Tiene una iniciativa y determinación en su trabajo. 
Además en el texto Alcaraz (2011), cita otras características que se le puede añadir y estas pertenecen a Burch (2008), y son las siguientes:

- Son trabajadores y tienen mucho afán por conseguir lo propuesto, es decir alcanzar las metas.

- Considera toda acción que es posible y sienten mucho optimismo por alcanzar los objetivos y metas.

- Tiene siempre a la excelencia es decir su deseo de lograr lo motiva a hacer mejor las cosas.

\section{Emprendedor florista}

El florista es una persona que se dedica a la producción y comercialización de flores, rosas, plantas en maceta y de cualquier otra forma de vender plantas sean estas en estado natural o artificial.

Dicho oficio pertenece a un sistema estructurado que se basa en el arte floral sea este de manera física, como también se tiene floristas virtuales que ofrecen plantas a través de sus páginas y entrega a domicilio.

Los floristas desarrollan su actividad en establecimientos o locales en donde exponen sus flores o plantas para que estos sean adquiridos por sus clientes, además no solo lo exponen en locales, sino que también lo hacen en kioskos, puestos de mercado o esquinas de las calles.

\section{Flor}

Una flor es una especie vegetal que tiene la función de reproducirse a través de sus semillas y así generar nuevas especies o nuevas plantas para ser utilizadas en distintas formas.

Según Domínguez (2016), "Es la estructura reproductiva característica de las plantas llamadas espermatofitas o fanerógamas, la función de una flor es producir semilla a través de la reproducción sexual."

Además "Para las plantas, las semillas son la próxima generación y sirven como el principal medio a través del cual las especies se perpetúan y se propagan.”.

\section{El color}

Según Paz (2016), el color de las flores se debe dos tipos de pigmentos, el primero que es conocido como pigmentos liposolubles y estos se encuentran presentes en los cromoplastos y el segundo son los pigmentos hidrosolubles contenidos en las vacuolas de las células epidérmicas de los pétalos: "La gran mayoría de tonos azules y purpuras se deben gracias a pigmentos vacuolares llamados antocianinas. Estos cambian de color en función del grado de acidez o alcalinidad del tipo exacto de antocianina: si la solución vacuolar es básica, el color es azul si es neutra, vira al purpura o al violeta".

\section{Flor cortada}

Se considera una flor cortada cuando esta tienen un tamaño, es decir su porte es apto para utilizarlas en arreglos florales o simplemente para regalarlas solas. Pero a su vez hay que tener 
en cuenta que estas flores no duran más de tres días cortadas y sin marchitarse, luego de este tiempo se morirán.

\section{Duración de las flores cortadas}

De acuerdo a lo que expone Salaz (2018), nos explica la duración que tiene cada flor de acuerdo a su corte.

- Son efímeras aquellas que viven entre 1 y 3 días. Entre estas encontramos a las Begonias, Peonias y Nenúfares.

- Son de corta duración debido a que viven de 3 a 5 días. Entre estas encontramos a los Tulipanes y a las violetas.

- Se las considera de tiempo medio pues una duración de entre 5 y 10 días, estas son utilizadas para hacer ramos, es decir aquí encontramos a las famosas rosas.

- Se la considera de larga duración pues estas flores viven entre 10 y 15 días. Entre ellos están los Gladiolos.

- Se las considera de gran duración puesto que viven de 15 días. Entre ellas están las Orquídeas, crisantemos.

\section{Materiales y Métodos.}

Los datos del artículo pertenecen a las floristerías de la ciudad de Riobamba, surgidas como emprendimiento. La investigación se desarrolló como un estudio transversal, descriptivo y analítico. La unidad de análisis son las 53 microempresas que se encuentran en las patentes catastradas del Gobierno Autónomo Descentralizado del cantón Riobamba, mismas que se encuentran activas. Las herramientas utilizadas para el desarrollo de este artículo fueron encuestas, entrevistas y observación directa a los comercios de la localidad dedicados a esta actividad. En la encuesta se aplicaron preguntas que determinan sus inicios y si clasifican como una microempresa, así como identificar ciertos problemas que no les permiten un desarrollo más eficiente. El alcance de la investigación es descriptiva, y la recolección de datos se efectuó en un momento único, es decir es un estudio observacional. Se aplico un cuestionario estructurado a los gerentes o responsables con mayor poder de decisión de la microempresa y que tenga información actual sobre la misma.

\section{Método del Muestro}

Para la elaboración de esta investigación se tomó en cuenta como universo a las microempresas de la ciudad de Riobamba que se dedican a la actividad motivo del estudio.

\section{Método de investigación basada en la opinión.}

El método de investigación basado en la opinión menciona que se debe hacer un estudio de manera aleatoria, con ayuda de las encuestas que están dirigidas a los microempresarios y así obtener datos tanto cualitativos como cuantitativos, que se podrán reunir gracias a la encuesta que se pretende aplicar.

\section{Resultados.}

Las floristerías de la ciudad de Riobamba tienen como actividad principal la realización de arreglos florales en especial para funerarias, es lo que más venden de acuerdo a lo que han 
comentado sin embargo sus arreglos también son demandados para ocasiones especiales como cumpleaños, día de la madre, día del maestro, navidad y para eventos en locales cerrados como: bodas, quinceaños, bautizos.

\section{Tabla 1 Matriz FODA Floristerías}

\section{FORTALEZAS}

$\checkmark \quad$ Ubicación estratégica del negocio

$\checkmark$ Dedicación y esmero por parte de los trabajadores

$\checkmark \quad$ Tendencia a la innovación

$\checkmark \quad$ Materia prima de alta calidad

$\checkmark$ Conocimiento sobre la actividad que se realiza

$\checkmark \quad$ Es un negocio familiar no se gasta en mano de obra

\section{OPORTUNIDADES}

$\checkmark$ Convenios con empresas que se dedican a la decoración de eventos

$\checkmark$ Fechas importantes las personas compran más flores

$\checkmark \quad$ Nuevas propuestas

$\checkmark \quad$ Las flores son cultivadas en el país no se necesita de importación

$\checkmark \quad$ El mercado es amplio

$\checkmark \quad$ Financiamiento por parte de entidades publicas

\section{DEBILIDADES}

$\checkmark \quad$ Las flores se marchitan con rapidez

$\checkmark \quad$ No se cuenta con clientes fijos

$\checkmark$ Se desconoce lo que el cliente busca

$\checkmark$ El personal no se encuentra motivado para realizar su trabajo

$\checkmark \quad$ No se cuenta con un horario accesible al cliente

$\checkmark \quad$ No se utiliza redes sociales para promocionar su negocio

$\checkmark \quad$ No se realizan entregas a domicilio de arreglos florales

\section{AMENAZAS}

$\checkmark \quad$ Diversos negocios que se dedican a la misma actividad (competencia)

$\checkmark \quad$ No lograr fidelidad de los clientes

$\checkmark$ Inseguridad y alta delincuencia

$\checkmark$ Competencia con flores artificiales

$\checkmark$ Plagas a los sembríos de la flores los precios suben

$\checkmark \quad$ El cliente busca precios económicos

$\checkmark \quad$ Los clientes prefieren otras decoraciones, ya no flores

Fuente: Investigación de mercado

Elaborado por: Los autores

La encuesta se realizó a 53 personas propietarias de las floristerías de la ciudad de Riobamba, mismas que fueron tabuladas y arrojaron los siguientes resultados.

Pregunta 1. ¿A qué se dedica su empresa?

Tabla 2 A qué se dedica su empresa

\begin{tabular}{lll}
\hline \multirow{2}{*}{ Respuesta } & Total & \\
\cline { 2 - 3 } & Cantidad & $\mathbf{\%}$ \\
\hline Producción de flores & 8 & $15 \%$ \\
Venta de flores & 45 & $85 \%$ \\
Total & $\mathbf{5 3}$ & $\mathbf{1 0 0 \%}$ \\
\hline
\end{tabular}

Fuente: Investigación de mercado

Elaborado por: Los autores 
Figura 1 A qué se dedica su empresa

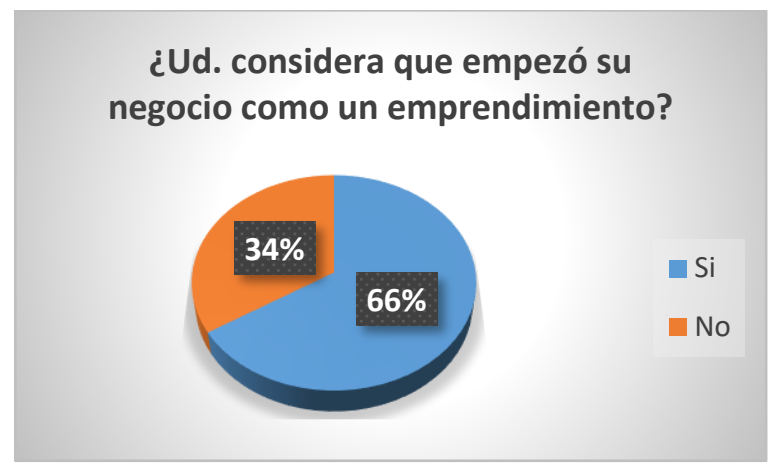

Fuente: Investigación de mercado

Elaborado por: Los autores

\section{Interpretación}

De la población de 53 personas el $85 \%$ de personas, es decir, 45 personas se dedican a la venta de flores y arreglos netamente siendo esta su actividad económica principal mientras que apenas el $15 \%$ de la población se dedica a la producción de flores pero estas personas también se dedican a la comercialización de las mismas.

Pregunta 2. ¿Ud. considera que empezó su negocio como un emprendimiento?

Tabla 3 Ud. considera que empezó su como un emprendimiento

\begin{tabular}{lll}
\hline Respuesta & $\begin{array}{l}\text { Total } \\
\text { Cantidad }\end{array}$ & $\mathbf{\%}$ \\
\cline { 1 - 2 } $\mathrm{Si}$ & 53 & $100 \%$ \\
No & 0 & $0 \%$ \\
Total & $\mathbf{5 3}$ & $\mathbf{1 0 0 \%}$ \\
\hline
\end{tabular}

Fuente: Investigación de mercado

Elaborado por: Los autores

Figura 2 Está asociado a otra persona

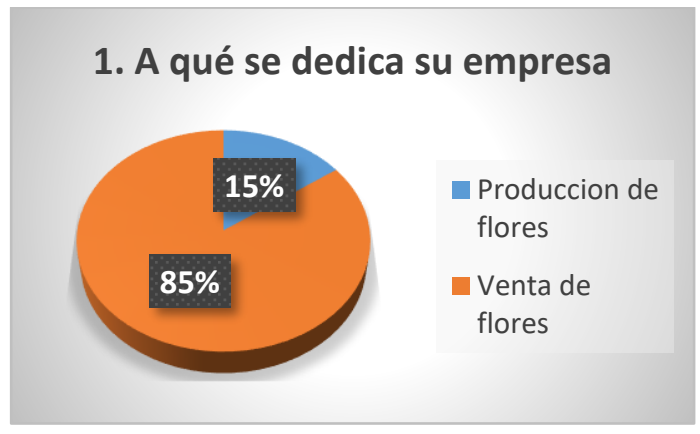

Fuente: Investigación de mercado

Elaborado por: Los autores 


\section{Interpretación}

En la pregunta de si los microempresarios del negocio de las flores empezaron como un emprendimiento la totalidad de los mismos afirman que si surgieron como un emprendimiento en base a la necesidad de generar ingresos para sus familias y algunos de los mismos son negocios que han pasado de generación en generación.

\section{Pregunta 3. ¿Con cuántos trabajadores cuenta su negocio?}

Tabla 4 Con cuántos trabajadores cuenta su negocio

\begin{tabular}{lll}
\hline \multirow{2}{*}{ Respuesta } & Total & \\
\cline { 2 - 3 } & Cantidad & $\mathbf{\%}$ \\
\hline 10 & 8 & $15 \%$ \\
9 & 15 & $28 \%$ \\
5 & 22 & $42 \%$ \\
Otros & 8 & $15 \%$ \\
Total & $\mathbf{5 3}$ & $\mathbf{1 0 0 \%}$ \\
\hline
\end{tabular}

Fuente: Investigación de mercado

Elaborado por: Los autores

Figura 3 Con cuántos trabajadores cuenta su negocio

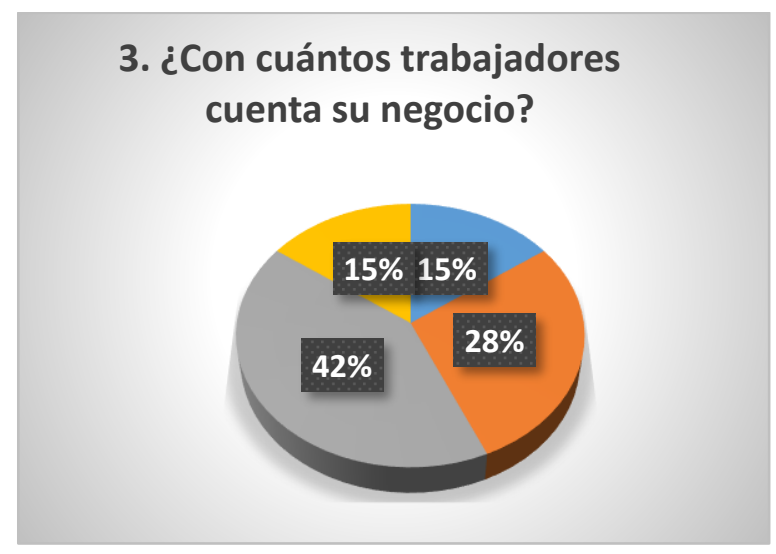

Fuente: Investigación de mercado

Elaborado por: Los autores

\section{Interpretación}

De la encuesta realizada a 53 personas cuya pregunta era con cuántos trabajadores cuenta su negocio 22 personas que representan el $42 \%$ respondieron que cuentas con 5 trabajadores, 15 personas el $28 \%$ respondieron que cuentan con 9 trabajadores, 8 personas el $15 \%$ respondieron que cuentan con 10 trabajadores y el mismo porcentaje respondieron que cuentan con menos de 5 trabajadores al ser estos negocios familiares trabajan los emprendedores junto con sus hijos no cuentan con demasiada mano de obra siendo un gasto menos y ayudándoles a mejorar sus utilidades 


\section{Pregunta 4. ¿Tienen otros locales?}

Tabla 5 Tiene otros locales

\begin{tabular}{lll}
\hline \multirow{2}{*}{ Respuesta } & Total & \\
\cline { 2 - 3 } & Cantidad & $\mathbf{\%}$ \\
\hline $\mathrm{Si}$ & 13 & $25 \%$ \\
$\mathrm{No}$ & 40 & $75 \%$ \\
Total & $\mathbf{5 3}$ & $\mathbf{1 0 0 \%}$ \\
\hline
\end{tabular}

Fuente: Investigación de mercado

Elaborado por: Los autores

Figura 4 Tienen otros locales

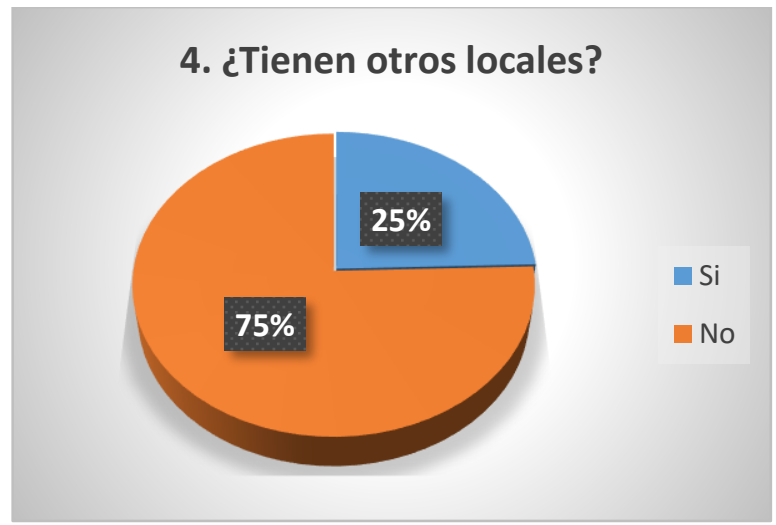

Fuente: Investigación de mercado

Elaborado por: Los autores

\section{Interpretación}

En la pregunta 6 en donde se preguntó si cuentan con otros locales la población contesto el $75 \%$, es decir, 40 personas respondieron que no que los gastos les permite contar con un solo local mientras que el $25 \%$, es decir 13 personas contestaron que si cuentan con otros locales que los ingresos que han alcanzado les ha permitido abrir sucursales que son manejadas en muchas ocasiones por la misma familia o que es manejado por la misma persona se traslada de un local a otros gastando menos en mano de obra

\section{Pregunta 5. ¿A qué tipo de clientes va enfocado el producto que venden?}

Tabla 6 A qué tipo de clientes va enfocado el producto que vende

\begin{tabular}{lll}
\hline \multirow{2}{*}{ Respuesta } & Total & \\
\cline { 2 - 3 } & Cantidad & $\mathbf{\%}$ \\
\hline Clase Baja & 15 & $28 \%$ \\
Clase Media & 25 & $47 \%$ \\
Clase Alta & 13 & $25 \%$ \\
Total & $\mathbf{5 3}$ & $\mathbf{1 0 0 \%}$ \\
\hline
\end{tabular}

Fuente: Investigación de mercado

Elaborado por: Los autores 
Figura 5 Ha qué tipo de clientes va enfocado su negocio

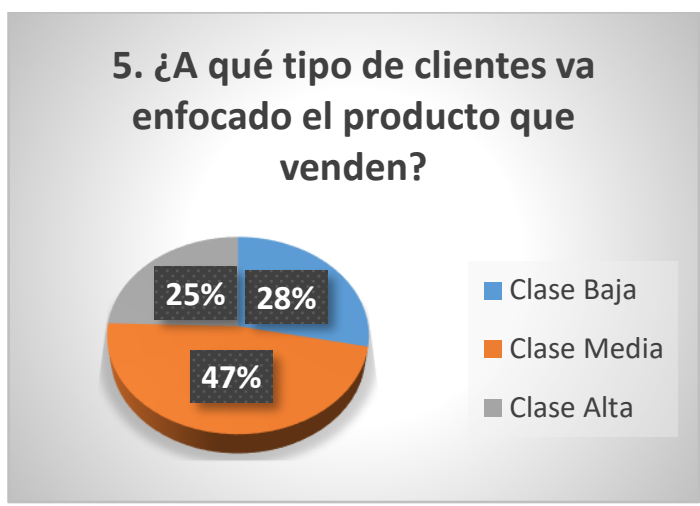

Fuente: Investigación de mercado

Elaborado por: Los autores

\section{Interpretación}

La mayoría de estos negocios están dedicados a la clase media un $47 \%$ afirma esto, mientras que el $28 \%$ afirma que sus productos están dirigidos a personas de clase baja y apenas $25 \%$ personas afirman que su negocio está dirigido a personas de clase alta, esto se debe a que al ofrecer precios económicos es más accesible para personas con una economía baja mientras personas de clase alta prefieren productos innovadores que están apareciendo en el mercado.

Pregunta 6. ¿Cuántas horas dedica usted a su negocio?

Tabla 7 Cuantas horas dedica usted a su negocio

\begin{tabular}{lll}
\hline Respuesta & $\begin{array}{l}\text { Total } \\
\text { Cantidad }\end{array}$ & $\mathbf{\%}$ \\
\cline { 3 - 3 } 15 horas & 12 & $23 \%$ \\
10 horas & 18 & $34 \%$ \\
8 horas & 15 & $28 \%$ \\
7 horas & 5 & $9 \%$ \\
Otros & 3 & $6 \%$ \\
Total & $\mathbf{5 3}$ & $\mathbf{1 0 0 \%}$ \\
\hline
\end{tabular}

Fuente: Investigación de mercado

Elaborado por: Los autores

Figura 6 Cuantas horas le dedica usted a su negocio

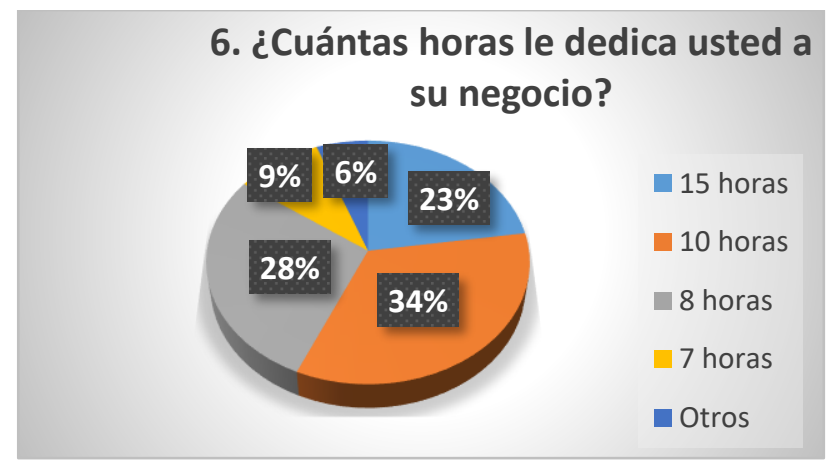

Fuente: Investigación de mercado

Elaborado por: Los autores 


\section{Interpretación}

Al ser emprendedores y empezar con este negocio como una forma para mejorar sus economías la mayoría de personas afirman que le dedican todo el tiempo posible a su negocio 18 personas manifiestan que le dedican entre 10 a más horas a su negocio representando el $34 \%$ de la población sin embargo el $23 \%$ de la población, es decir, 12 personas dicen que le dedican más de 15 horas a su negocio; el $28 \%$ le dedica a su negocio 8 horas; el $9 \%$ le dedica únicamente 7 horas a su negocio y apenas el 6\% le dedica menos de 7 horas pues afirman que cuentan con otros negocios que les impide dedicarse por completo a las floristerías

\section{Pregunta 7. ¿Con qué tipo de proveedores cuenta usted?}

Tabla 8 Con qué tipo de proveedores cuenta usted

\begin{tabular}{lll}
\hline \multirow{2}{*}{ Respuesta } & Total & \\
\cline { 2 - 3 } & Cantidad & $\mathbf{\%}$ \\
\hline Nacionales & 24 & $45 \%$ \\
Locales & 29 & $55 \%$ \\
Total & $\mathbf{5 3}$ & $\mathbf{1 0 0 \%}$ \\
\hline
\end{tabular}

Fuente: Investigación de mercado

Elaborado por: Los autores

Figura 7 Con qué tipo de proveedores cuenta usted

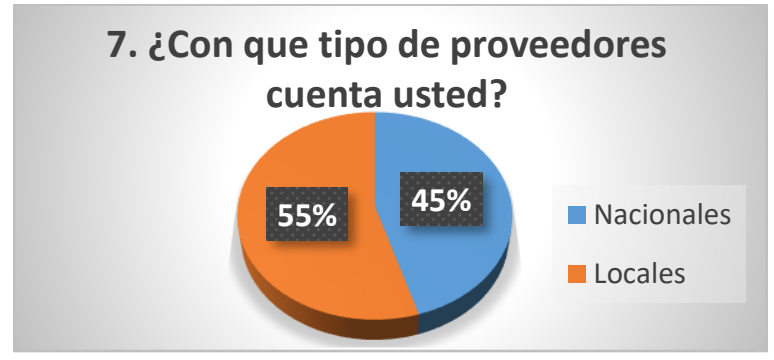

Fuente: Investigación de mercado

Elaborado por: Los autores

\section{Interpretación}

Las flores son un producto que se encuentra a nivel nacional al realizar la pregunta de con qué tipo de proveedores cuenta usted 29 personas, es decir, el 55\% afirman que sus principales proveedores son locales ya que ofrecen productos más económicos que los proveedores nacionales mientras que 24 personas manifiestan que sus proveedores son nacionales ya que estos ofrecen materia prima de calidad permitiéndoles brindar un producto terminado de mejor calidad que satisfaga las expectativas del cliente

Pregunta 8. ¿Quién es su competidor más cercano? 
Tabla 9Quién es su competidor más cercano

\begin{tabular}{lll}
\hline \multirow{2}{*}{ Respuesta } & Total & \\
\cline { 2 - 3 } & Cantidad & $\mathbf{\%}$ \\
\hline Floristería Aledañas & 47 & $89 \%$ \\
Floristerías & & \\
Reconocidas & 6 & $11 \%$ \\
Total & $\mathbf{5 3}$ & $\mathbf{1 0 0 \%}$ \\
\hline
\end{tabular}

Fuente: Investigación de mercado

Elaborado por: Los autores

Figura 8 Quién es su competidor más cercano

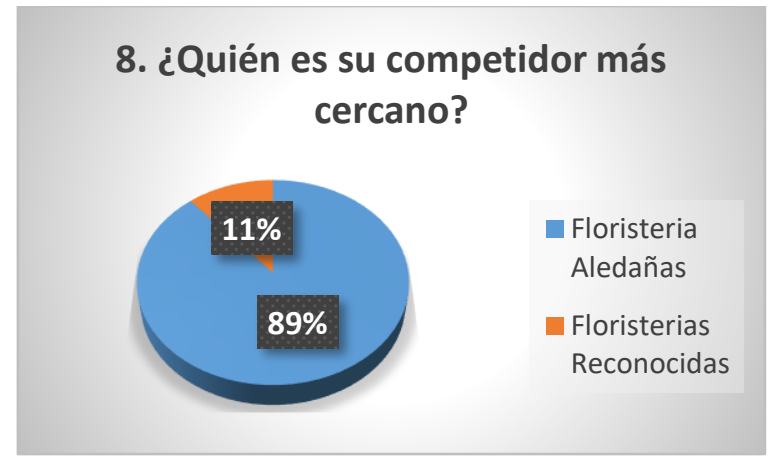

Fuente: Investigación de mercado

Elaborado por: Los autores

\section{Interpretación}

Las florerías están ubicadas en una zona estratégica también cuentan con demasiada competencia por tal razón a la pregunta de ¿Quién es su competidor más cercano? Fue casi unánime la respuesta de que su competidor más fuerte son las florerías aledañas esto es lo que afirman 47 personas es decir un 89\% de la población y apenas 6 personas que representan el $11 \%$ afirman que su competencia son las floristerías reconocidas sin embargo la mayoría de la población manifiesta que en la ciudad no se cuenta con una gran cantidad de floristería reconocida por lo que no lo ven como una competencia real.

Pregunta 9. ¿Cuenta con personal externo que le ayude en el negocio?

Tabla 10Cuenta con personal externo que le ayude en el negocio

\begin{tabular}{lll}
\hline & Total & \\
\cline { 2 - 3 } Respuesta & Cantidad & $\mathbf{\%}$ \\
\hline Contador & 15 & $28 \%$ \\
Publicista & 12 & $23 \%$ \\
Marketing & 5 & $9 \%$ \\
Abogado & 5 & $9 \%$ \\
Ninguno & 16 & $30 \%$ \\
Total & $\mathbf{5 3}$ & $\mathbf{1 0 0 \%}$ \\
\hline
\end{tabular}

Fuente: Investigación de mercado

Elaborado por: Los autores 
Figura 9 Cuenta con personal externo que le ayude en el negocio

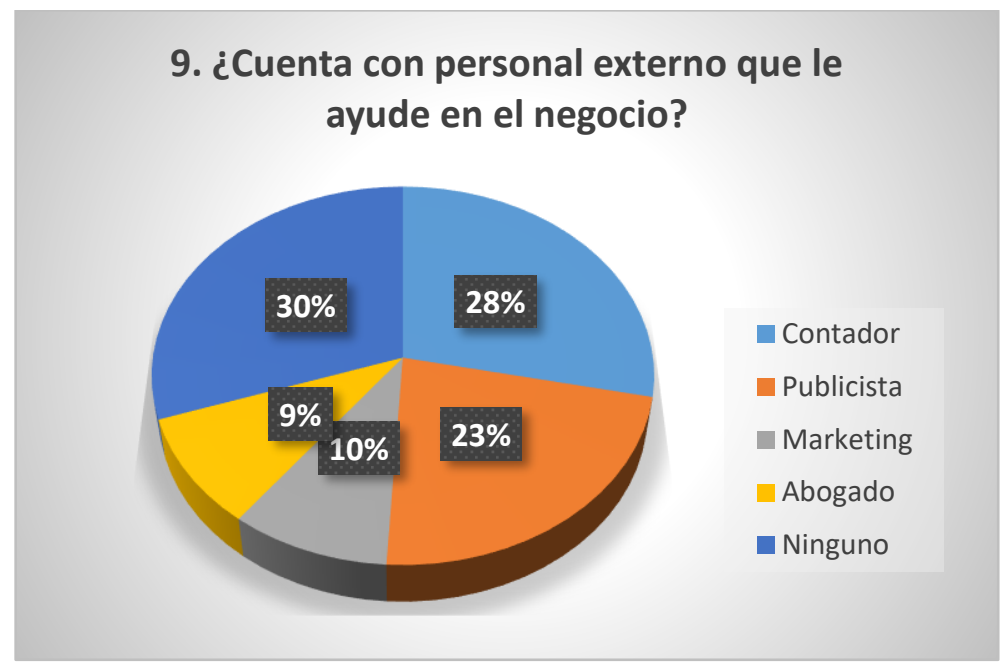

Fuente: Investigación de mercado

Elaborado por: Los autores

\section{Interpretación}

De acuerdo a la actividad económica las floristerías se han venido manejando de una manera empírica, sin embargo dicen que en la actualidad necesitan de la asesoría de personal capacitado por ello cuando se realizó la pregunta de si contaban con personal externo que le ayude en el negocio, 15 personas afirman que cuentan con la asesoría de un contador por tema de tributos y de si están realizando de manera correcta la contabilidad 12 personas que representan un $23 \%$ de la población han implementado un publicista para atraer mayor cantidad de clientes a su negocio el $10 \%$ cuenta con alguien con conocimientos en marketing y apenas el $9 \%$ cuenta con la asesoría de un abogado para su negocio mientras que el $30 \%$ establece que no necesitan la asesoría de personal externo que como lo han estado haciendo hasta ahora les ayudado y les ha ido bien entonces no se requiere la presencia de personas extrañas.

Pregunta 10. ¿De acuerdo al giro de su emprendimiento, Ud. consideraría que hoy es una microempresa?

Tabla 111Cuenta con personal externo que le ayude en el negocio

\begin{tabular}{lll}
\hline \multirow{2}{*}{ Respuesta } & Total & \\
\cline { 2 - 3 } & Cantidad & $\mathbf{\%}$ \\
\hline $\mathrm{Si}$ & 35 & $66 \%$ \\
$\mathrm{No}$ & 18 & $34 \%$ \\
Total & $\mathbf{5 3}$ & $\mathbf{1 0 0 \%}$ \\
\hline
\end{tabular}

Fuente: Investigación de mercado

Elaborado por: Los autores 
Figura 10 Cuenta con personal externo que le ayude en el negocio

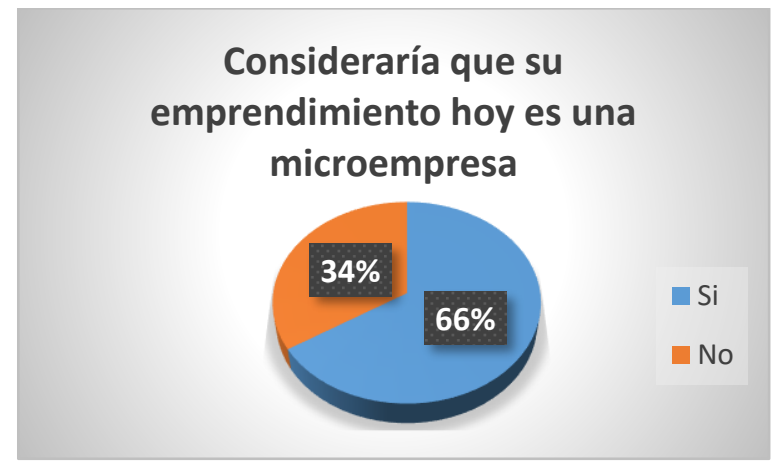

Fuente: Investigación de mercado

Elaborado por: Los autores

\section{Interpretación}

Según los resultados obtenidos en esta pregunta se puede observar que un gran porcentaje considera que en la actualidad su emprendimiento es una microempresa.

\section{Discusión}

Según los resultados encontrados a través de la aplicación de encuestas al sector de las microempresas comercializadoras de flores de la cuidad de Riobamba hemos podido determinar que entre los resultados determinantes de la investigación se determinó que la totalidad de comercios surgieron como emprendimientos y además el $66 \%$ de los encuestados manifiestan que sus negocios se podrían considerar como microempresas.

También se pudo determinar ciertas consideraciones que tienen las floristerías de la ciudad, los emprendimientos no cuentan con una ayuda técnica en el estudio de negocio, la mayor competencia son las floristerías aledañas, esto se debe a que existe la presencia de funerarias en dicho sector y esto provoca que se hayan incrementado en estos últimos años decir de ellos, también han manifestado no contar con la contribución o apoyo de algún socio o profesional en el área jurídica, publicitaria, contabilidad, lo que determina que estos negocios simplemente fueron creados básicamente como un emprendimiento,

Sin embargo estos negocios son fuente generadora de dinero para la cuidad, pues están destinados a cualquier segmento económico, por lo que por esta razón permite que cualquier ciudadano tenga la posibilidad de adquirirlo lo que de acuerdo a su giro económico manifiestan que ellos se consideran como una microempresa y además se encuentran creciendo.

\section{Conclusión}

- Los emprendimientos de la ciudad de Riobamba surgieron como un emprendimiento, generados por la necesidad de concebir ingresos económicos para llevar el sustento a sus hogares, De la población de 53 personas el 85\% de personas, es decir, 45 personas se dedican a la venta de flores y arreglos netamente siendo esta su actividad económica principal mientras que apenas el 15\% de la población se dedica a la producción de flores pero estas personas también se dedican a la comercialización de las mismas, los microempresarios del negocio de las flores empezaron como un emprendimiento en 
base a la necesidad de generar ingresos para sus familias y algunos de los mismos son negocios que han pasado de generación en generación.

- A estos emprendimientos se les dedica una gran cantidad de horas de trabajo, lo que ha permitido que los mismos sean sostenibles y que los propietarios les consideren como microempresas, La mayoría de estos negocios están enfocados hacia a la clase media un $47 \%$ afirma esto, mientras que el $28 \%$ afirma que sus productos están dirigidos a personas de clase baja y apenas $25 \%$ personas afirman que su negocio está dirigido a personas de clase alta, esto se debe a que al ofrecer precios económicos es más accesible para personas con una economía baja mientras personas de clase alta prefieren productos innovadores que están apareciendo en el mercado.

\section{Referencias Bibliográficas}

Alcaraz, R. (2011). Emprendedor de Éxito . Mexico : McGRAW-HILL/INTERAMERICANA EDITORES,

Ameconi, O. (2004). Microempresas en Acción. Buenos Aires: MACCHI.

Borras, f., Fernadez, A., \& Martinez, F. (2017). El emprendimiento: una aproximacion internacional al desarrollo economico. Santander: Universidad Cantabria.

Dominguez. (01 de 05 de 2016). Bitacora Verde. Obtenido de Bitacora Verde Blogspot.com: http://bitacoraverde01.blogspot.com/2016/05/la-flor-es-la-estructurareproductiva.html

Marchant, C. (12 de 06 de 2017). Camaleo. Obtenido de Camaleo: https://es.calameo.com/read/0012900445ce326f8db9a

Paz, R. (26 de 08 de 2016). EcuRed. Obtenido de EcuRed: https://webcache.googleusercontent.com/search?q=cache:BHx7KrJzFawJ:https://ww w.ecured.cu/Flor $+\& \mathrm{~cd}=1 \& \mathrm{hl}=\mathrm{es}-419 \& \mathrm{ct}=\mathrm{clnk} \& \mathrm{gl}=\mathrm{ec}$

Salaz, C. (23 de 05 de 2018). El hogar Natural . Obtenido de El hogar Natural : http://www.elhogarnatural.com/reportajes/flor\%20cortada.htm

Schnarch, A. (2014). Emprendimiento exitosocomo mejorar su proceso y gestión . Bogota : Eco ediciones.

Telégrafo. (28 de 06 de 2017). El 95\% de las empresas en el país son mipymes. El Telegrafo, pág. 27.

Urbano, D., \& Toledano, N. (2008). Invitacion al emprendimiento una aproximacion a la creacion de empresas. Barcelona: UOC. 


\section{PARA CITAR EL ARTÍCULO INDEXADO.}

Vallejo Sánchez, D. P., Colcha Ortiz, R. V., \& Moreno Albuja., M. del C. (2020). Las floristerías, microempresas generadas como emprendimientos en base a la normativa legal. ConcienciaDigital, 3(2.2), 33-49. https://doi.org/10.33262/concienciadigital.v3i2.2.1244

\section{\Ciencia}

El artículo que se publica es de exclusiva responsabilidad de los autores y no necesariamente reflejan el pensamiento de la Revista Conciencia Digital.

El artículo queda en propiedad de la revista y, por tanto, su publicación parcial y/o total en otro medio tiene que ser autorizado por el director de la Revista Conciencia Digital.
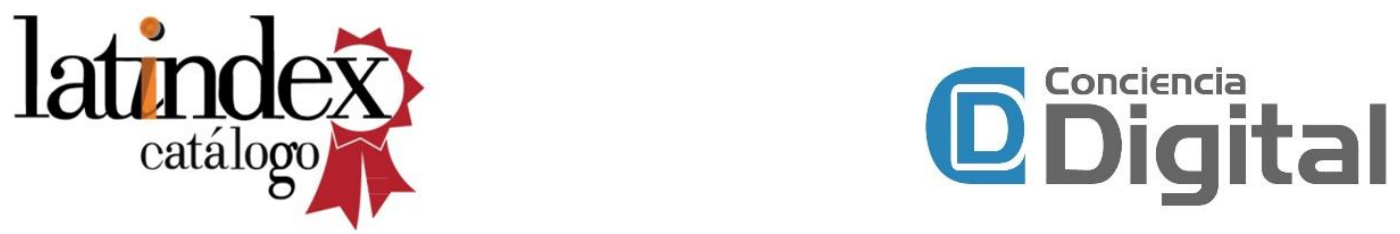